\title{
Characterizing posets for which their natural transit functions coincide*
}

\author{
Boštjan Brešar \\ Faculty of Natural Sciences and Mathematics, University of Maribor, Slovenia \\ Manoj Changat \\ Department of Futures Studies, University of Kerala, Trivandrum-695 034, India \\ Sandi Klavžar \\ Faculty of Mathematics and Physics, University of Ljubljana, Slovenia \\ and \\ Faculty of Natural Sciences and Mathematics, University of Maribor, Slovenia \\ Joseph Mathews \\ Chingamparampil, Vazhapally, Changanacherry, 686103 Kerala, India \\ Antony Mathews \\ Department of Futures Studies, University of Kerala, Trivandrum-695 034, India \\ Prasanth G. Narasimha-Shenoi \\ Department of Mathematics, Government College, Chittur, Palakkad-678 104, India
}

Received 11 July 2008, accepted 19 December 2008, published online 4 March 2009

\begin{abstract}
The standard poset transit function of a poset $P$ is a function $T_{P}$ that assigns to a pair of comparable elements the interval between them, while $T_{P}(x, y)=\{x, y\}$ for a pair $x, y$ of incomparable elements. Posets in which the standard poset transit function coincides with the shortest-path transit function of their cover-incomparability graph are characterized in three ways, in particular with forbidden subposets.
\end{abstract}

Keywords: Transit function, ranked poset, underlying graph, geodesic interval, induced-path interval. Math. Subj. Class.: 05C12, 05C62, 06A07

\footnotetext{
*Work supported by the Ministry of Science of Slovenia and by the Ministry of Science and Technology of India under the bilateral India-Slovenia grants BI-IN/06-07-002 and DST/INT/SLOV-P-03/05, respectively.

E-mail addresses: bostjan.bresar@uni-mb.si (Boštjan Brešar), mchangat@gmail.com (Manoj Changat), sandi.klavzar@fmf.uni-lj.si (Sandi Klavžar), jose_chingam@yahoo.co.in (Joseph Mathews), sonykandans@yahoo.co.in (Antony Mathews), gnprasanth@gmail.com (Prasanth G. Narasimha-Shenoi)
} 


\section{Introduction}

The notion of convexity has been extended from Euclidean spaces to several different mathematical structures. In the last several decades an abstract theory of convex structures was developed. It is based on just two (or three in the infinite case) natural conditions, imposed to a family of subsets of a given set. The theory is comprehensively surveyed in van de Vel's book [13], where the so-called interval convexity turns out as one of its universal concepts.

As shown by Calder in [3], the pertinent properties of a mapping $I: X \times X \rightarrow 2^{X}$ that yields a convexity on a set $X$ are the symmetry law $(I(x, y)=I(y, x)$ for all $x, y \in X)$ and the extensive law $(x, y \in I(x, y))$. These two laws constitute all axioms for the formal interval convexity with $I$ as the interval operator [13]. By adding also the idempotent law $(I(x, x)=\{x\}$ for all $x \in X)$, one gets the concept of a transit function as defined by Mulder [10]. His purpose was to generalize geodesic (and some other) intervals in graphs and other discrete structures, including posets. Furthermore, for a set $X$ on which a transit function $T$ is defined, the underlying graph $G_{T}$ was introduced as the graph with $X$ as its vertex set, where distinct $u$ and $v$ in $X$ are adjacent whenever $|T(u, v)|=2$.

In a poset $P=(X, \leq)$ by the notion of interval $[x, y]$ one usually assumes that $x$ and $y$ are two comparable elements, and $[x, y]=\{z: x \leq z \leq y\}$. This function can be extended to all pairs of elements in a poset, by setting $T_{P}(x, y)=\{x, y\}$ if $x$ and $y$ are incomparable, and $T_{P}(x, y)=[x, y]$ otherwise $[7,10,13]$. This yields an interval convexity in the general sense as described above. Clearly $T_{P}$ is also a transit function on $P$ and is called the standard poset transit function.

The underlying graph $G_{T_{P}}$ of the standard poset transit function $T_{P}$ was studied in [1] under the name cover-incomparability graph of a poset $P$. (For other connections between posets and graphs we refer to [12].) In $G_{T_{P}}$ the most common transit function is given by the geodesic intervals (formed by vertices on shortest paths between pairs in $V\left(G_{T_{P}}\right)$ ), which is denoted $I_{G_{P}}$. The natural question appears, in which posets both transit functions (interval convexities) $T_{P}$ and $I_{G_{P}}$ coincide. In this note we characterize such posets in two ways, once by listing forbidden subposets, and in the other case as certain maximal ranked posets. Interestingly, in such posets also the induced-path transit function $J_{G_{P}}$ in the underlying graph coincides with the standard poset transit function of $P$, which is, in fact, characteristic for these posets.

In the next section we fix terminology and state some simple observations. In Section 3 we prove the main theorem. In the conclusion we show how the theorem can be extended from finite to chain-finite, countable posets.

\section{Preliminaries}

A transit function on a non-empty set $V$ is a function $T: V \times V \rightarrow 2^{V}$ satisfying the following axioms:

(t1) $u \in T(u, v)$ for any $u$ and $v \in V$.

(t2) $T(u, v)=T(v, u)$ for all $u$ and $v \in V$.

(t3) $T(u, u)=\{u\}$ for all $u \in V$.

Prominent examples of transit functions on a connected graph $G=(V, E)$ are the geodesic transit function (studied extensively in [9]):

$$
I_{G}(u, v)=\{w \in V \mid w \text { lies on a shortest } u, v \text {-path in } G\},
$$


the induced-path transit function [5, 8]:

$$
J_{G}(u, v)=\{w \in V \mid w \text { lies on an induced } u, v \text {-path in } G\},
$$

and the all-paths transit function $A$, which is the coarsest path transit function on $G[4,6]$ :

$$
A_{G}(u, v)=\{w \in V \mid w \text { lies on some } u, v \text {-path in } G\} .
$$

Recall $[2,11]$ that a ranked poset is a poset $P=(V, \leq)$ that is equipped with a rank function $\rho: V \rightarrow \mathbb{Z}$ satisfying:

- $\rho$ is constant on all minimal elements of $P$ (usually with value -1 or 0 ), and

- $\rho$ preserves covering relations: if $b$ covers $a$ then $\rho(b)=\rho(a)+1$.

A ranked poset $P$ is said to be complete if for every $i$, every element of rank $i$ covers all the elements of rank $i-1$.

Let $P=(V, \leq)$ be a poset. Then the poset $Q=\left(V^{\prime}, \leq^{\prime}\right)$ is a subposet of $P$ if $V^{\prime} \subseteq V$ and if for any elements $u$ and $v$ of $V^{\prime}, u \leq^{\prime} v$ if and only if $u \leq v$.

Recall that the height of a poset $P$ is the maximum size of a chain in $P$, and a poset is called bipartite if its height is at most 2 [2].

Let $P=(V, \leq)$ be a poset, then the graph $G_{P}=(V, E)$ is called the cover-incomparability graph of $P$ if $a b \in E$ whenever $a$ covers $b$, or $b$ covers $a$, or $a$ and $b$ are incomparable. In other words, the edge set of $G_{P}$ is the union of the edge set of the cover graph of $P$ and the incomparability graph of $P$. Note that the cover-incomparability graph $G_{P}$ of a poset $P$ is just the underlying graph of the standard poset transit function. Hence $G_{T_{P}}$ would be an appropriate notation for the cover-incomparability graph (as stated in the introduction) which we will shorten to $G_{P}$ in the sequel. We denote by $I_{G_{P}}$ the geodesic transit function in the cover-incomparability graph $G_{P}$, and $J_{G_{P}}$ the induced-path transit function in $G_{P}$.

It can be easily verified that for any poset $P$, the graph $G_{P}$ is connected [1]. Let $u, v$ be a pair of incomparable elements of a poset $P$. If $P$ has more than two elements, then it is clear that there exists another element $z$ in $P$ which either covers or is incomparable with one of $u$ or $v$ or both $u$ and $v$. In all cases $A_{G_{P}}(u, v)$ (where $A_{G_{P}}$ stands for the all-paths transit function in $\left.G_{P}\right)$ contains $z$, but $T_{P}(u, v)$ consists only of $u$ and $v$ alone. Therefore, we have the following observation:

Remark 2.1. Let $P=(V, \leq)$ be a poset on at least three elements. Then $T_{P}=A_{G_{P}}$ if and only if $P$ is a chain.

\section{The theorem}

In this section we characterize the posets $P=(V, \leq)$ in which $T_{P}=I_{G_{P}}$. First, if $P$ is bipartite (that is, the size of a longest chain is at most 2), then $G_{P}$ is a complete graph which readily implies:

Remark 3.1. Let $P=(V, \leq)$ be a bipartite poset. Then $T_{P}=I_{G_{P}}=J_{G_{P}}$.

In the following theorem we consider the non-trivial case, when the height of a poset is greater than 2 .

Theorem 3.2. Let $P=(V, \leq)$ be a poset of height at least 3 . Then the following statements are equivalent: 
(i) $T_{P}=I_{G_{P}}$;

(ii) $T_{P}=J_{G_{P}}$;

(iii) $P$ has no subposet isomorphic to $P_{1}, P_{2}$, or $P_{3}$, see Figure 1;

(iv) $P$ is a complete ranked poset.

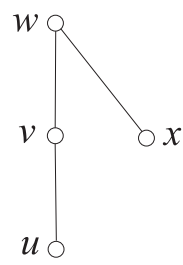

$P_{1}$

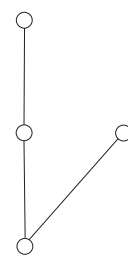

$P_{2}$

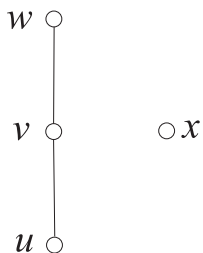

$P_{3}$

Figure 1: Forbidden subposets.

In the rest of this section we prove Theorem 3.2 and begin with the following implication.

$(i) \Rightarrow($ iii $)$. Let $u, v, w, x$ be the elements of $P$ that form a subposet isomorphic to $P_{1}$, see Figure 1. Let $x=x_{0}, x_{1}, \ldots, x_{k}=w$ be a maximal $x, w$-chain in $P$. Note that $k \geq 1$, and that if $k=1$ then $w=x_{1}$. Note also that none of the elements $x_{i}, i<k$, is below $v$ because otherwise $x$ would be below $v$.

Let $i, 1 \leq i \leq k$, be the smallest index such that $v \leq x_{i}$. Such an index exists since $v \leq w=x_{k}$. Then $v$ and $x_{i-1}$ are incomparable. Let $v=v_{0}, v_{1}, \ldots, v_{j}=x_{i}$ be a maximal $v, x_{i}$-chain in $P$. Note that $j \geq 1$.

Suppose $j \geq 2$. Then consider $v_{j-2}, v_{j-1}, v_{j}, x_{i-1}$. We claim that $v_{j-2}$ is incomparable with $x_{i-1}$. Indeed, if $x_{i-1} \leq v_{j-2}$ held, then $x_{i}$ would not be covering $x_{i-1}$. And if $v_{j-2} \leq x_{i-1}$ we would have $v \leq x_{i-1}$, which is not possible by the selection of $i$. Analogous argument implies that $v_{j-1}$ is incomparable with $x_{i-1}$. Now observe that $d_{G_{P}}\left(v_{j-2}, x_{i}\right)=2$ hence $x_{i-1} \in I_{G_{P}}\left(v_{j-2}, x_{i}\right)$. On the other hand, $x_{i-1} \notin T_{P}\left(v_{j-2}, x_{i}\right)$, a contradiction.

Suppose $j=1$, that is, $x_{i}$ covers $v$. If $i \geq 2$ then $v \in I_{G_{P}}\left(x_{i-2}, x_{i}\right)$ but $v \notin$ $T_{P}\left(v_{i-2}, x_{i}\right)$. Let $i=1$. Then $x_{1}$ covers $x$. Let $y$ be a vertex on a $u, v$-chain that is covered by $v$. Then $y \leq x$ would imply $u \leq x$. Moreover, $x \leq y$ would mean that $x$ is not covered by $x_{1}$. It follows that $y$ and $x$ are incomparable and hence $x \in I_{G_{P}}\left(y, x_{1}\right)$ but $x \notin T_{P}\left(y, x_{1}\right)$.

Thus we have proved that if $P$ has a subposet $P_{1}$ then $T_{P} \neq I_{G_{P}}$. Analogous argument also yields that a subposet $P_{2}$ implies that $T_{P} \neq I_{G_{P}}$.

Assume finally that $u, v, w, x$ are the elements of $P$ that form a subposet isomorphic to $P_{3}$, see Figure 1 . Let $u=u_{0}, u_{1}, \ldots, u_{k}=w$ be a $u, w$-chain that contains $v$. Then $k \geq 2$ and moreover, none of the elements $u_{i}$ is comparable to $x$. Then $x \in I_{G_{P}}\left(u, u_{2}\right)$ but $x \notin T_{P}\left(u, u_{2}\right)$.

$($ ii $) \Rightarrow($ iii $)$. This implication follows by the same arguments as the implication $(i) \Rightarrow(i i i)$ because a path of length 2 is a shortest path if and only if it is induced.

$($ iii $) \Rightarrow($ iv $)$. Assume that $P$ has no subposet isomorphic to $P_{1}, P_{2}$, or $P_{3}$. Let $C=u_{0} \leq$ $u_{1} \leq \cdots \leq u_{n}$ be a longest chain in $(P, \leq)$. Then $n \geq 2$. 
Define $S_{0}$ as the set consisting of the minimal elements of P. Further define $S_{i}, 1 \leq i \leq$ $n$, as the subset of $V$ containing $u_{i}$ and the elements of $P$ which are incomparable with $u_{i}$ and that cover $u_{i-1}$.

Claim 1. $u_{0} \in S_{0}$.

This is clear because otherwise $C$ would not be a longest chain.

Claim 2. Elements of $S_{i}, 0 \leq i \leq n$, are pairwise incomparable.

This is clear for $i=0$. Consider different elements $v_{i}$ and $w_{i}$ of $S_{i}, i \geq 1$. By the definition of $S_{i}$, both $v_{i}$ and $w_{i}$ cover $u_{i-1}$. But then $v_{i}$ and $w_{i}$ are incomparable.

Claim 3. For $i \geq 1$ any element of $S_{i}$ covers every element of $S_{i-1}$.

Assume first $i \geq 2$ and consider elements $v_{i} \in S_{i}$ and $w_{i-1} \in S_{i-1}$. Since $v_{i} \in S_{i}$, it covers $u_{i-1}$. By Claim 2, $u_{i-1}$ and $w_{i-1}$ are incomparable and hence $w_{i-1}$ cannot be below $u_{i-2}$. If $v_{i}$ and $w_{i-1}$ are incomparable, then $v_{i}, u_{i-1}, u_{i-2}, w_{i-1}$ form an induced $P_{2}$. Hence $v_{i}$ and $w_{i-1}$ are comparable. Using Claim 2 again, $w_{i-1}$ cannot be above $v_{i}$ hence there must be a chain $w_{i-1}<x \leq \cdots \leq v_{i}$.

Suppose $x \neq v_{i}$. Then $x$ and $u_{i-1}$ are incomparable. Indeed, $u_{i-1} \leq x$ would yield $u_{i-1} \leq x \leq v_{i}$, a contradiction with the fact that $v_{i}$ covers $u_{i-1}$. On the other hand, $x \leq u_{i-1}$ would give us that $w_{i-1} \leq x \leq u_{i-1}$ contradicting Claim 2. So $x$ and $u_{i-1}$ are incomparable, but then $v_{i}, x, w_{i-1}, u_{i-1}$ induce $P_{1}$. We conclude that $x=v_{i}$ and hence $v_{i}$ covers $w_{i-1}$. Note that the same argument also applies if $v_{i}=u_{i}$.

It remains to prove Claim 3 for $i=1$. Let $v_{1} \in S_{1}$ and $w_{0} \in S_{0}$ be arbitrary elements. Then $v_{1}$ covers $u_{0}$. By the above paragraph, $u_{2}$ covers $v_{1}$. If $w_{0}$ and $v_{1}$ are incomparable, then $u_{0}, v_{1}, u_{2}, w_{0}$ induce $P_{3}$ or $P_{1}$. Since $w_{0}$ is a minimal element, we can thus only have $w_{0} \leq v_{1}$. Let $x$ be the first element on a maximal $w_{0}, v_{1}$-chain. Then we can argue as above that $x=v_{1}$.

Claim 4. $\bigcup_{i=0}^{n} S_{i}=V$.

Let $v$ be an arbitrary element of $P$. Consider a maximal chain $v_{0} \leq v_{1} \leq \cdots \leq v_{m}$ in $P$ that contains $v$. Clearly, $m \leq n$. Hence $v=v_{i}$ for some $0 \leq i \leq m$. We claim that $v \in S_{i}$ and prove it by induction. The element $v_{0}$ is minimal, hence $v_{0} \in S_{0}$. Assume now that $v_{i-1} \in S_{i-1}$. Hence by Claim 3, $u_{i}$ covers $v_{i-1}$. As $v_{i}$ also covers $v_{i-1}$, it follows that $u_{i}$ and $v_{i}$ are incomparable. If $u_{i-1}$ and $v_{i}$ are incomparable then consider $u_{i}$, $u_{i-1}, u_{i-2}, v_{i}$ (or, if $i=1$, consider $u_{i-1}, u_{i}, u_{i+1}, v_{i}$ ) to obtain $P_{2}$ or $P_{3}$ (or, $P_{1}$ or $P_{3}$ ). So $u_{i-1}$ and $v_{i}$ are comparable and it is only possible that $u_{i-1} \leq v_{i}$. Consider a chain $u_{i-1} \leq x \leq v_{i}$. Then $x$ and $v_{i-1}$ are incomparable. But then $u_{i-1}, x, v_{i}, v_{i-1}$ induce $P_{1}$. Hence $x=v_{i}=v$. Therefore $v$ covers $u_{i-1}$ and since in addition $v_{i}$ is incomparable to $u_{i}$ we conclude that $v=v_{i} \in S_{i}$.

Claim 5. For $0 \leq j \leq i-2 \leq n-2$, no element of $S_{i}$ covers an element of $S_{j}$.

Let $v_{i} \in S_{i}, w_{j} \in S_{j}, j \leq i-2$. Suppose $v_{i}$ covers $w_{j}$. Let $v_{j} \leq v_{j+1} \leq \cdots \leq v_{i}$, where $v_{k} \in S_{k}$, be a $v_{j}, v_{i}$-chain. Then $w_{j}$ is incomparable with $v_{j}, \ldots, v_{i-1}$. Hence $v_{i-2}, v_{i-1}, v_{i}, w_{j}$ induce a $P_{1}$, a contradiction that proves Claim 5 .

For $v \in V$, define $\rho: V \rightarrow \mathbb{Z}$ by $\rho(v)=i$ where $v \in S_{i}$. Then $\rho$ is a rank function on $P$. Moreover, Claim 3 implies that this ranked poset is complete.

To complete the proof note that implications $(i v) \Rightarrow(i)$ and $(i v) \Rightarrow(i i)$ are straightforward. 


\section{Extension to infinite posets}

Let $P=(V, \leq)$ be an arbitrary poset. Then $P$ is called chain-finite if every chain between any comparable elements $u$ and $v$ is finite. We claim that Theorem 3.2 extends to countable, chain-finite posets with the following modifications.

By a complete ranked poset we now mean a poset whose elements can be partitioned into sets $S_{i}, i \in \mathbb{Z}$, such that every element of $S_{i}$ covers every element of $S_{i-1}$ for all $i$ and that $S_{k} \neq \emptyset$ as soon as there are indices $i$ and $j$ such that $i<k<j$ and $S_{i} \neq \emptyset, S_{j} \neq \emptyset$.

Let $P=(V, \leq)$ be a countable, chain-finite poset. Then note that $G_{P}$ is a connected (infinite) graph and hence $I_{G_{P}}$ and $J_{G_{P}}$ are well-defined.

With these additions Theorem 3.2 holds for such posets. The proof for $(i) \Rightarrow(i i i)$ and $(i i) \Rightarrow(i i i),(i v) \Rightarrow(i)$ and $(i v) \Rightarrow(i i)$ goes along the same lines as in the finite case. To prove $(i i i) \Rightarrow(i v)$ we can define the sets $S_{i}$ in a similar way. Notably, let $C$ be an arbitrary maximal chain of $P$ and let its elements be $\ldots, u_{-2}, u_{-1}, u_{0}, u_{1}, u_{2}, \ldots$ (or $u_{0}, u_{1}, u_{2}, \ldots$ if $P$ has minimal elements, and $\ldots, u_{-2}, u_{-1}, u_{0}$ if $P$ has maximal elements). Then define $S_{i}, i \in \mathbb{Z}$, as the subset of $V$ containing $u_{i}$ and the elements of $P$ which are incomparable with $u_{i}$ and cover $u_{i-1}$.

Now in the proof of $(i i i) \Rightarrow(i v)$ most of the claims go through regardless of finiteness of $P$. More precisely, Claim 1 is the same, or even not needed if $P$ has no minimal and no maximal elements (if $P$ has only maximal elements then an analogous trivial claim has to be used). Claims 2 and 5 have the same proofs as above, while Claim 3 is even easier in the case when $P$ has no minimal elements, since the last paragraph of its proof is not needed. Hence, we only need to prove Claim 4 which is the only non-trivial part of the extension to infinite posets.

Let $x$ be an arbitrary element of a countable, chain-finite poset $P$. We need to show that there exists an $i$ such that $x \in S_{i}$ (from this one finds that $\bigcup_{i} S_{i}=V$ ). The first step is to show that $x$ is incomparable with exactly one element from $\ldots, u_{-2}, u_{-1}, u_{0}, u_{1}, u_{2}, \ldots$

Suppose that $x$ is incomparable with $u_{i}$ and $u_{j}$, and let $i<j$. Then $u_{i}, u_{j}, u_{j+1}$ and $x$ form a subposet isomorphic to $P_{1}$ or $P_{3}$, a contradiction. Next, suppose $x$ is comparable with all elements of the maximal chain $C$. We claim that then the set $S=\left\{u_{i} \in C: u_{i} \leq\right.$ $x\}$ is nonempty and bounded from above. Suppose this is not the case. Then assuming that $S$ is empty (resp. $S$ not bounded from above) we quickly derive that for all $u_{i} \in C$ we have $x \leq u_{i}$ (resp. $u_{i} \leq x$ ), which is a contradiction with $C$ being a maximal chain. Thus, let $u_{j}$ be the largest element in $C$ such that $u_{j} \leq x$, and note that $u_{j}$ cannot be a maximal element in $P$ because $C$ is a maximal chain. Thus $x \leq u_{j+1}$ which again contradicts the fact that $C$ is maximal since $x$ can be inserted between $u_{j}$ and $u_{j+1}$, extending the chain $C$. Hence $x$ cannot be comparable to all elements of $C$ either.

Thus $x$ is incomparable with exactly one element of $C$, say $u_{i}$. If $u_{i}$ is a minimal element then $i=0$ and $x \in S_{0}$. Hence, let $u_{i}$ not be minimal, and we need to show that $x$ covers $u_{i-1}$ (this will imply that $x \in S_{i}$ ).

It is clear that $u_{i-1} \leq x \leq u_{i+1}$. Let $v$ be the first element on a chain from $u_{i-1}$ to $x$, and assume that $v \neq x$. Suppose that $v$ is incomparable with $u_{i}$. Then, since $x$ is also incomparable with $u_{i}$ we infer that $u_{i-1}, u_{i}, v$ and $x$ form a subposet, isomorphic to $P_{2}$. Since this is forbidden, the only possibility is that $v$ and $u_{i}$ are comparable. If $u_{i} \leq v$, then by transitivity $u_{i} \leq x$, but $u_{i}$ and $v$ are incomparable. Also $v \leq u_{i}$ is not possible, since then one could extend the chain $C$ between $u_{i-1}$ and $u_{i}$ with $v$ and the chain between $v$ and $u_{i}$. Since $C$ is a maximal chain this is a contradiction, hence $v=x$. Thus $x$ covers $u_{i-1}$ and so $x \in S_{i}$. Theorem 3.2 indeed extends to countable chain-finite posets. 


\section{References}

[1] B. Brešar, M. Changat, S. Klavžar, M. Kovše, J. Mathews and A. Mathews, Cover-incomparability graphs of posets, Order 25 (2008), 335-347.

[2] G. Brightwell and D. B. West, Partially ordered sets, in: K.H. Rosen (ed.), Handbook of Discrete and Combinatorial Mathematics, CRC Press, Boca Raton, 2000, 717-752.

[3] J. R. Calder, Some elementary properties of interval convexities, J. London Math. Soc. 3 (1971), 422-428.

[4] M. Changat, S. Klavžar and H. M. Mulder, The all path transit function of a graph, Czech. Math. J. 51 (2001), 439-448.

[5] M. Changat and J. Mathews, Induced path transit function, monotone and Peano axioms, Discrete Math. 286 (2004), 185-194.

[6] M. Changat, H. M. Mulder and G. Sierksma, Convexities related to path properties on graphs, Discrete Math. 290 (2005), 117-131.

[7] A. Mathews and J. Mathews, Transit functions on posets and lattices, in: M. Changat, S. Klavžar, H. M. Mulder and A. Vijayakumar (eds.), Convexity in Discrete Structures, Lecture Notes Ser. 5, Ramanujan Math. Soc., 2008, 105-116.

[8] M. A. Morgana and H. M. Mulder, The induced path convexity, betweenness and svelte graphs, Discrete Math. 254 (2002), 349-370.

[9] H. M. Mulder, The Interval Function of a Graph, Mathematisch Centrum, Amsterdam, 1980.

[10] H. M. Mulder, Transit functions on graphs (and posets), in: M. Changat, S. Klavžar, H. M. Mulder, A. Vijayakumar (eds.), Convexity in Discrete Structures, Lecture Notes Ser. 5, Ramanujan Math. Soc., 2008, 117-130.

[11] D. Stanton, D. White, Constructive Combinatorics, Springer, New York, 1986.

[12] W. Trotter, Graphs and partially ordered sets: recent results and new directions, Cong. Numer. 116 (1996), 253-278.

[13] M. van de Vel, Theory of Convex Structures, North-Holland, Amsterdam, 1993. 